\title{
Deporte y homosexualidad: Revisión literaria en bases de datos científicas iberoamericanas con senvicio de open access
}

Sport and homosexuality: Scoping review in ibero-american scientific databases with open access service

Ciria Margarita Salazar C.

Universidad de Colima, México

ciria6@ucol.mx

(iD) https://orcid.org/0000-0001-8863-2309

Emilio Gerzain Manzo Lozano

Universidad de Colima, México

manzolozano@ucol.mx

(iD https://orcid.org/0000-0003-2087-2378

Patricia Sánchez Espinosa

Asociación 100 por Colima, AC, México

patricia.sanez@gmail.com

(iD) https://orcid.org/0000-0002-4117-415X

\section{Resumen:}

El presente texto hace una revisión sistemática de alcance sobre lo publicado en bases de datos científicas iberoamericanas con sistema open Access (acceso abierto) de la temática deporte y homosexualidad. La técnica a utilizar es la revisión sistemática de alcance también llamada scoping review basada en la metodología del Instituto Joanna Briggs: fuentes consultadas, criterios de inclusión y exclusión, estrategia de búsqueda, tamización y selección de estudios, extracción de datos, análisis y presentación de resultados. Entre los hallazgos y resultados más relevantes encontramos: la heterogeneidad como estilo de práctica y vida; la discriminación y necesidades de los grupos LGBTTT; estereotipos por el tipo de práctica deportiva; experiencias de vida en el deporte; activismo mediante el deporte y el trabajo inconcluso de la educación física en temas de diversidad sexual.

Palabras Clave: Deporte, Homosexualidad y revisión sistemática de alcance.

\begin{abstract}
:
This text aims at making a bibliographic review that addresses issues related to sports and homosexuality and sexual diversity. It results from a documentary analysis in the most widely used Spanish-speaking Open Access databases in Latin America (Latin America and Spain). The most recurring problems in the studies addressed are: heterogeneity as a style of practice and life, discrimination and needs of LGBTTT groups, stereotypes for the type of sports practice, life experiences in sports, activism through sport, and the unfinished work of Physical Education on issues of sexual diversity.
\end{abstract}

KEYWORDS: Sports, Homosexuality and Sexual diversity.

\section{INTRODUCCIÓN}

El ámbito deportivo se asocia a la visión de masculinidad, fuerza, rapidez, competitividad, liderazgo, organización jerárquica y supremacía. El origen del mismo, surge en un espacio androcéntrico y heteronormativo (Piedra et. al, 2014) que buscaba a través del deporte fortalecer el espíritu de la raza humana y reflejar los valores del marco moral-cultural de las naciones (Blanchard \& Cheska,1986). Fue Thomas 
Arnold quien introduce al deporte como un elemento educativo para producir voluntades firmes y corazones rectos, al mismo tiempo que cuerpos robustos; mediante la libertad y la jerarquía del mérito; y su gran discípulo, Pierre de Coubertin, en la carta olímpica de 1918 refiere a uno de los beneficios de la promoción del deporte y los juegos olímpicos modernos al ejercicio físico masculino como agente de compensación orgánica y el ejercicio físico como creador de fuerza moral y fuerza nacional (Pérez-Aragón \& Gallardo-Pérez, 2016).

Queda claro que el deporte nació masculino y la apertura al otro sexo y a los otros géneros ha sido lenta y polémica, mayormente en el Comité Olímpico Internacional (COI) máxima organización mundial del deporte (Sagarzazu \& Lallana, 2012). En cuanto al sexo femenino, las mujeres se involucraron en el deporte organizado y oficial en Paris 1900; pero es en 1924 cuando se vuelve oficial la presencia en Juegos Olímpicos. Sin embargo, esa incorporación se logró primeramente en deportes considerados femeninos o adecuados a ellas (López Díaz, 2011 en Sagarzazu \& Lallana, 2012) como la gimnasia, el tenis o la natación, para el 2016, las mujeres estaban en todas las disciplinas deportivas, excepto en beisbol en el programa olímpico.

En 1968 el COI exigió pruebas de verificación de género hasta Sidney 2000, ya que ninguna contenía la precisión científica biológica para definir el sexo de los deportistas. Relata Juny González Garzón (2019) que el análisis de la participación de intersexuales y transexuales tuvo eco en el máximo organismo apenas 20 años, la autora, del texto Educación Física y deporte trans-formado, relata que:

El 28 de octubre de 2003, un comité especial designado por el COI y una comisión médica se reunió para analizar la posible participación de personas que modificaran su sexo, y fue hasta el 27 de mayo de 2004 que se les permitió la participación para los Juegos Olímpicos de Pekín de 2008, con ciertos requisitos: Cambios anatómicos quirúrgicos finalizados, reconocimiento legal de sexo asignado y terapia de reemplazo hormonal realizada de manera comprobable y con tiempo suficiente para minimizar las ventajas relacionadas con el género en competiciones deportivas.

Sin embargo, las críticas sociales y prejuiciadas y la falta de investigación han alargado la incorporación y reconocimiento por completo del COI y la comunidad mundial; esto ha generado, en 2015 modificaciones para permitir la participación diferenciada de deportistas hombres y mujeres trans.

Las cuestiones que refieren a la individualidad del deportista deberían no interferir en su desenvolvimiento social, pero el panorama ante los medios, en un perímetro de reconocimiento regional o internacional, el factor de las preferencias sexuales permea la figura del deportista a partir elementos sociales fundamentales: La personalidad del deportista en tanto líder y modelo ante los cánones, ritos y costumbres provenientes del modelo heterosexual -en tanto objeto de referencia, desde la visión de Baudrillard (2004) que establece: "el hombre de colocación" [...] dispone del espacio como de una estructura de distribución; a través del control de este espacio, dispone de todas las posibilidades de relaciones recíprocas y, por tanto, de la totalidad de los papeles que pueden desempeñar los objetos. El deportista impone una ubicación en el espacio social.

Los diferentes movimientos incluyentes que surgen desde manifestaciones sociales, políticas y artísticas alrededor del mundo para buscar la eliminación de la homofobia y otras prácticas exclusivas que afectan la sana convivencia entre los seres humanos. Muchos son los documentos, surgidos desde desde organismos internacionales, que proponen la incorporación de la inclusión y la equidad en los sistemas educativos de los países con la finalidad de generar un cambio que se refleje en términos de acceso, participación, procesos y resultados del aprendizaje dentro de una mejor calidad de vida y, por lo tanto, en una manifestación plena de la persona independientemente de sus características particulares (Organización de las Naciones Unidas para la Educación, la Ciencia y la Cultura, 2017).

La Organización de las Naciones Unidas (ONU) propone que todas las personas tienen derecho al goce de sus derechos humanos sin distinción alguna de raza, color, sexo, idioma, religión, opinión política o de cualquier otra índole, origen nacional o social, posición económica, nacimiento o cualquier otra condición, tal como lo establecen el artículo 2 de la Declaración Universal de los Derechos Humanos y el artículo 2 de los Pactos Internacionales de Derechos Civiles y Políticos y de Derechos Económicos, Sociales y Culturales, así como el artículo 26 del Pacto Internacional de Derechos Civiles y Políticos. El principio de 
no discriminación, que exige que los derechos humanos se apliquen por igual a todos los seres humanos, independientemente de su orientación sexual o identidad de género (ONU, 2008).

En el caso del deporte, el tema trans e inter comenzó a ser importante para el COI en 1950 con la comprobación de la intersexualidad de una multimedallista de atletismo. De eso han pasado 71 años, y aun la discusión sigue dando lugar al prejuicio, a la falta de conocimiento científico y reconocimiento social. Esto da origen a luchas de activistas pro-deporte trans, erigiendo sus propios espacios de participación y pujando en diversos países para ampliar las posibilidades desde la jurisprudencia, la ciencia, la investigación y la deconstrucción de la cultura hegemónica.

\section{Los estudios deporte y diversidad sexual}

Es justo desde la ciencia en donde los conocimientos han venido abriéndose camino con nuevas aristas y miradas al descubrimiento y aceptación de otras realidades más allá de las ciencias básicas. Barreiro (2004) menciona que el progreso científico genera soluciones técnicas que cambian el significado del cuerpo natural (medio del deporte), en otras palabras, ya dicho por Giddens (1995) surge "la socialización de la naturaleza", lo que era aceptado solamente desde una visión naturalista, hoy con los adelantos en campos sociales, la constante en las agendas de investigación y divulgación ayuda a construir oportunidades de equidad y justicia, y progreso en esos campos invisibilizados.

La divulgación de los resultados del trabajo intelectual encuentra salidas en libros, revistas, ponencias y hasta en comunicaciones informales. Sin embargo, las revistas especializadas siguen siendo el medio de comunicación más utilizado por los investigadores (Salazar, Arellano, Pérez y Manzo, 2014) para continuar cultivando líneas de investigación en su búsqueda de crear o renovar posiciones sobre temas científicos.

En el ámbito de las ciencias sociales, la perspectiva de la sociología del deporte, busca constantemente explicaciones sobre los comportamientos del movimiento deportivo, para explicar, reflexionar y encontrar nuevas formas de recrear o entender al deporte de hoy.

García Ferrando (1990:15-16) en Moscoso (2006) plantea que existen al menos tres formas de acercarse al estudio del deporte desde la perspectiva sociológica.

1. Es la de carácter experimental, que consiste en emplear el fenómeno deportivo para contrastar proposiciones generales relacionadas con la conducta social.

2. La que trata de comprender este fenómeno haciendo uso de los conceptos, las teorías y los métodos propios de la sociología.

3. Es la de la perspectiva fenomenológica, entendida como una manera singular de afrontar el estudio del deporte, caracterizada por ocuparse de la comprensión de los significados que para los actores tiene la práctica de esta actividad, es decir, de aquellos rasgos que distinguen o caracterizan a este fenómeno social. "Muchos autores que siguen esta linea contemplan el deporte como una subcultura propia, con sus especificas normas y reglas, e incluso con su propio funcionamiento" (García Ferrando, 1990 en Moscoso, 2006: 179).

En este sentido, los estudios sociológicos del deporte son diversos y ricos en propuestas ideológicas, corrientes, sucesos y metodologías; por ello, se busca, abonar refiriendo a la divulgación de los estudios relacionados con la homosexualidad en el deporte; está por demás, justificar, que la divulgación de nuevos conocimientos fomenta la inclusión y tratamiento a tópicos socialmente complejos de explicar para ciertas comunidades, más aquellas, que no tienen apego mayor a la ciencia que a las religiones; de igual forma, la divulgación, impide crear caos e incertidumbre en las sociedades, incluso les permite tomar decisiones con mayor propensión al éxito. 
El presente texto, busca mostrar una panorámica sobre la publicación, visibilidad y avances en los temas que refieren a homosexualidad y deporte; si bien, los estudios de género se han fortalecido en los últimos años, algunos temas han quedado rezagados como es el caso de la diversidad sexual (Kozak, 2006) y en lo relativo a sociología del deporte, existen escasos materiales.

Kozak, en el 2006, expresaba la invisibilidad de las universidades para abordar estos temas, expresaba que muchas no decidían salir del closet, para iniciar líneas de estudios o programas de investigación para las llamadas minorías sexuales.

En este mismo sentido mencionaba Barbero (2003) en Piedra (2019) poco se ha trabajado el tema, hasta estas dos últimas épocas que el abordaje ha resultado no ser un tabú, mucho de ese trabajo desde la perspectiva sociológica y pedagógica, y muy recientemente, desde la perspectiva de género.

Por ello, la presente revisión sistemática pretende, reconocer y ofrecer una panorámica de lo que la comunidad académica ha difundido en las Bases de Dato de acceso abierto, enfatizando en la combinación de los términos de búsqueda y no aislados (Pérez, Arellano \& Peña, 2013) sobre homosexualidad y deporte.

\section{Metodología}

La metodología que sigue el trabajo, es de revisión sistemática de alcance (scoping review) sobre lo publicado en bases de datos científicas iberoamericanas con sistema open Access (acceso abierto) de la temática deporte y homosexualidad. Manchado Garabito, Tamames, López, Mohedano, D 'Agostino \& Veiga de Cabo (2009) refiere a la scoping review como un proceso que permiten explorar no sólo qué se ha hecho sobre un tema, también quién, dónde y de qué manera, a partir de los resultados obtenidos pueden generarse hipótesis sobre futuras preguntas de investigación y proponerse ámbitos de estudio que no están suficientemente desarrollados.

La técnica a utilizar es la revisión sistemática de alcance también llamada scoping review basada en la metodología del Instituto Joanna Briggs: fuentes consultadas, criterios de inclusión y exclusión, estrategia de búsqueda, tamización y selección de estudios, extracción de datos, análisis y presentación de resultados (Fernández-Sánchez, King \& Enríquez-Hernández, 2020).

Las fuentes consultadas fueron las bases de datos Open Access de habla hispana más utilizadas o con mayor presencia en Iberoamérica (América Latina y España); siendo estás, Dialnet, Redalyc, Scielo, Latindex y Google Escolar en Español, este último utilizado como parámetro comparativo con las bases de datos abiertas y Science Research, de acceso con paga o en espacios universitarios. Siendo los criterios de inclusión y exclusión, primordialmente que fue fueran textos de plataformas o repositorios indizados en las bases de datos mencionadas, que se pudieran descargar de forma completa, que se encontraran publicadas entre 1998-2019, que abordaran las temáticas, deporte, homosexualidad y/o diversidad sexual, que fueran tesis, artículos, capítulos de libro, reseñas bibliográficas y/o ponencias (aprobadas por comités científicos).

Para las búsquedas especializadas en dichos repositorios, se utilizaron 14 combinaciones utilizando los operadores booleanos, con los términos deporte- homosexualidad AND diversidad sexual AND gays AND lesbianas AND transgénero AND bisexual, al ser, el futbol uno de los deportes mayormente practicados en el mundo y por su presencia hegemónica en los rituales de masculinización, se optó por buscar una combinación en tre futbol AND homosexualidad. De igual manera se realizaron combinaciones de búsqueda con Educación Física y Actividad Física (Ver Tabla 1) AND homosexualidad AND diversidad sexual AND gays AND lesbianas AND transgénero AND bisexual, encontrándose únicamente 20 documentos (tesis, artículos, capítulos de libro, reseña bibliográfica y ponencias) que hablan sobre el tema en cuestión de 1998-2019. Google Escolar y Science Research nos permitió confirmar la presencia de los textos y localización en las bases de datos indizadas; considerando que Google Escolar es un buscador y Science Research un repositorio que permite acceder libremente a las publicaciones que los autores suben a sus expedientes curriculares. 
Es importante mencionar que el trabajo de búsqueda bibliográfica ser realizó entre los meses de noviembre y diciembre de 2019. Hoy en día, la búsqueda refiere a más documentos que aparecen de los años 2020 y 2021, con nuevos abordajes. Sin embargo, el estudio refiere a dos décadas de análisis.

\section{TABLA 1}

Resultados de la combinación de búsquedas y selección de documentos en bases de datos y repositorios latinoamericanos 1998-2019

\begin{tabular}{|c|c|c|c|c|c|c|}
\hline Combinación de búsqueda & Dialnet & Redalyc & Scielo & Latindex & $\begin{array}{l}\text { Science } \\
\text { Reserch* }\end{array}$ & $\begin{array}{l}\text { Google } \\
\text { Escolar: }\end{array}$ \\
\hline Deporte y homosexualidad & 5 & 1 & 1 & 0 & 2 & 5 \\
\hline Deporte y lesbianas & 1 & 1 & 0 & 0 & 2 & 7 \\
\hline Deporte y gays & 1 & 0 & 0 & 0 & 1 & 3 \\
\hline Deporte y transgénero & 2 & 1 & 0 & 0 & 1 & 2 \\
\hline Deporte y bisexualidad & 0 & 0 & 0 & 0 & 1 & 0 \\
\hline Educación fisica y homosexualidad & 3 & 1 & 0 & 0 & 1 & 3 \\
\hline Educación fisica y lesbianas & 0 & 0 & 0 & 0 & 0 & 6 \\
\hline Educación fisica y gays & 0 & 0 & 0 & 0 & 0 & 3 \\
\hline Educación fisica y transgénero & 1 & 0 & 0 & 0 & 1 & 2 \\
\hline Educacion fisica y bisexualidad & 0 & 0 & 0 & 0 & 0 & 0 \\
\hline Actividad fisica y homosexualidad & 0 & 0 & 0 & 0 & 0 & 2 \\
\hline Actividad fisica y lesbianas & 0 & 0 & 0 & 0 & 0 & 1 \\
\hline Actividad fisica y gays & 0 & 0 & 0 & 0 & 0 & 0 \\
\hline Actividad fisica y transgenero & 1 & 0 & 0 & 0 & 1 & 1 \\
\hline Actividad fisica y bisexualidad & 0 & 0 & 0 & 0 & 0 & 0 \\
\hline
\end{tabular}

Fuente: Elaboración propia.

${ }^{*}$ Google Escolar y Science Research no se contabilizan como base de datos sino

\section{Resultados y Discusiones}

La revisión sistemática de alcance en diversas bases de datos de acceso abierto sobre deporte y homosexualidad muestran tendencias que nos hacen identificar el estado de la cuestión en dicha línea de generación del conocimiento. Es una realidad que los estudios sobre diversidad sexual son aún incipientes (Kozak, 2006), apenas encontramos en BD científicas de habla hispana 20 textos que han pasado por la revisión de pares, académicos de comités de expertos o árbitros externos para publicarse de 1998-2019 (Ver Tabla 2). 
TABLA 2

Recursos bibliográficos referenciados en las bases de datos y repetición en Index.

\begin{tabular}{|c|c|}
\hline $\begin{array}{l}\text { Base Datos/ } \\
\text { Repositorio }\end{array}$ & Texto \\
\hline \multirow{7}{*}{$\begin{array}{l}\text { DIALNET } \\
\qquad(\mathrm{n}=7)\end{array}$} & Homosexualidad y deporte, ¿un matrimonio mal avenido? (Piedra, 2015a) \\
\hline & $\begin{array}{l}\text { Ocio y homosexualidad un estudio etnográfico sobre el asociativismo deportivo de } \\
\text { mujeres, en el contexto de un deporte dicho masculino (Stigger \& } \mathrm{Da} \text { Silveira, 2010). }\end{array}$ \\
\hline & $\begin{array}{l}\text { Visibilizando lo invisible: creencias del profesorado de educación fisica sobre homofobia } \\
\text { y masculinidades (Piedra, Macías \& Romero, 2014). }\end{array}$ \\
\hline & $\begin{array}{l}\text { Percepción de los estudiantes sobre comportamientos homofóbicos y heterosexistas en } \\
\text { educación física (Silva, Jaeger, Valdivia-Moral, 2018). }\end{array}$ \\
\hline & $\begin{array}{l}\text { Entre queerpos e discursos: normalização de condutas, homossexualidades e homofobia } \\
\text { nas práticas escolares da Educação Física (Vagner, 2017). }\end{array}$ \\
\hline & $\begin{array}{l}\text { Educación fisica y deporte en personas Trans: una aproximación cualitativa (Fuentes, } \\
\text { 2015) }\end{array}$ \\
\hline & Transgénero y deporte (Garrido \& Collado, 2018). \\
\hline \multirow{4}{*}{$\begin{array}{l}\text { REDALYC } \\
(n=4)\end{array}$} & $\begin{array}{l}\text { Ocio y homosexualidad un estudio etnográfico sobre el asociativismo deportivo de } \\
\text { mujeres, en el contexto de un deporte dicho masculino (Stigger \& Da Silveira, 2010). }\end{array}$ \\
\hline & $\begin{array}{l}\text { Gays y lesbianas en el deporte: discurso de jóvenes universitarios españoles en tomo a su } \\
\text { aceptación(Piedra, 2015b). }\end{array}$ \\
\hline & $\begin{array}{l}\text { Entre queerpos e discursos: normalização de condutas, homossexualidades e homofobia } \\
\text { nas práticas escolares da Educação Física (Vagner, 2017) }\end{array}$ \\
\hline & $\begin{array}{l}\text { Escala de Actitudes hacia la Diversidad Sexual en el Deporte (EDSD): desarrollo y } \\
\text { validación preliminar (Piedra, 2016). }\end{array}$ \\
\hline $\begin{array}{l}\text { SCIELO } \\
(\mathrm{n}=1)\end{array}$ & $\begin{array}{l}\text { Ocio y homosexualidad un estudio etnográfico sobre el asociativismo deportivo de } \\
\text { mujeres, en el contexto de un deporte dicho masculino (Stigger \& Da Silveira, 2010). }\end{array}$ \\
\hline
\end{tabular}




\begin{tabular}{|c|c|}
\hline \multirow{5}{*}{$\begin{array}{l}\text { SCIENCE } \\
\text { RESEARCH } \\
(\mathrm{n}=5)\end{array}$} & $\begin{array}{l}\text { Ocio y homosexualidad un estudio etnográfico sobre el asociativismo deportivo de } \\
\text { mujeres, en el contexto de un deporte dicho masculino (Stigger \& Da Silveira, 2010). }\end{array}$ \\
\hline & $\begin{array}{l}\text { Prejuicio contra homosexuales en el contexto de fütbol (Pereira, Alfaia, Souza \& Lima, } \\
\text { 2014). }\end{array}$ \\
\hline & $\begin{array}{l}\text { Sexualidad y futbol. Dos resistencias femeninas en "Lesbianas de Buenos Aires" } \\
\text { (Antúnez, 2009). }\end{array}$ \\
\hline & Educación fisica y deporte en personas Trans: una aproximación cualitativa \\
\hline & $\begin{array}{l}\text { Soccer and homosexuality: The conflicts that lie within the affective game of the coach- } \\
\text { adolescent athlete relationship (Moioli, Machado, Zanetti, Campbell \& Gomez, 2014). }\end{array}$ \\
\hline \multirow{13}{*}{$\begin{array}{l}\text { GOOGLE } \\
\text { ESCOLAR } \\
(n=14)\end{array}$} & Fútbol y homosexualidad-un deporte para machos (Rodríguez, 1998). \\
\hline & $\begin{array}{l}\text { Ocio y homosexualidad un estudio etnográfico sobre el asociativismo deportivo de } \\
\text { mujeres, en el contexto de un deporte dicho masculino (Stigger \& Da Silveira, 2010). } \\
\text { Gays y lesbianas en el deporte: discurso de jóvenes universitarios españoles en tomo a su } \\
\text { aceptación }\end{array}$ \\
\hline & Homosexualidady deporte, ¿un matrimonio mal avenido? (Piedra, 2015a) \\
\hline & $\begin{array}{l}\text { Queering, el terreno de juego y salir a jugar: Lesbianas en la educación física y el deporte } \\
\text { (Clarke, 1998) }\end{array}$ \\
\hline & $\begin{array}{l}\text { Educación fisica y deporte en personas Trans: una aproximación cualitativa (Fuente, } \\
\text { 2015). }\end{array}$ \\
\hline & $\begin{array}{l}\text { Mujeres que "sexían" con mujeres. Experiencia sexual y deportiva entre futboleras de } \\
\text { Morelos (Ramírez, 2014). }\end{array}$ \\
\hline & $\begin{array}{l}\text { Cuerpos transexuales en las Olimpiadas: las políticas del Comité Intemacional Olímpico } \\
\text { en relación con l@s atletas transexuales en los Juegos de Verano, Atenas } 2004 \\
\text { (Cavanagh, Sykes \& Lamas, 2009). }\end{array}$ \\
\hline & $\begin{array}{l}\text { Reproducción de una parte: la vida de los profesores de educación física lesbianas } \\
\text { (Clarke, 1997) }\end{array}$ \\
\hline & $\begin{array}{l}\text { A Educação Física como território de demarcação dos gêneros possíveis: vivências } \\
\text { escolares de pessoas travestis, transexuais e transgêneros (Franco, 2016) }\end{array}$ \\
\hline & "Coligay" y la diversidad sexual en el ámbito deportivo (De Sousa \& De Camargo, 2015) \\
\hline & $\begin{array}{l}\text { La voz del cuerpo, prejuicios de la vista y práctica deportiva. Expresiones corpóreas y } \\
\text { mitos sobre la homosexualidad(Salazar \& Manzo, 2016). }\end{array}$ \\
\hline & $\begin{array}{l}\text { El colectivo LGTBI en el deporte como objeto de investigación sociológica. Estado de la } \\
\text { cuestión (Moscoso \& Piedra, 2019). }\end{array}$ \\
\hline & $\begin{array}{l}\text { Abriendo amarios. Reportaje ciberperiodístico sobre la realidad de los deportistas } \\
\text { homosexuales en España (Pontes de Los Ríos, 2019). }\end{array}$ \\
\hline
\end{tabular}


El país con mayor producción de textos es España, seguido de Brasil (Ver Tabla 3); ambos países, representan académicamente una tradición en varias LGAC (Líneas de generación y aplicación del conocimiento) de las ciencias sociales, especialmente en los estudios de género y de diversidad sexual. De igual forma, ambos países, dan muestra de tener investigadores con la formación y definición de LGAC orientadas a los estudios sobre diversidad sexual, que permiten consolidar rutas de investigación, usar teorías e ir generando un estado de conocimiento sobre el tema. En lo relativo al resto de los países latinoamericanos, México y Argentina, han publicado apenas dos documentos en índex de Open Access.

Sin embargo, el panorama aun para el abordaje de estos temas es hostil, Piedra (2019) observa progreso por varios académicos tras el análisis y propuesta para observar al deporte más allá de una visión dicotómica de hombre o mujer, lo que esperanza, que en la próxima década exista un mayor crecimiento de la investigación con perspectiva de género y la generación de posiciones teóricas, que ayuden a generar intervenciones y reflexiones incluyentes.

El análisis también visibiliza estudios con poblaciones y muestras muy definidas. Son las mujeres deportistas lesbianas en quienes se han hecho más estudios; seguidos, de las intervenciones con alumnos y docentes de los niveles de educación básica. La presencia de estudios con lesbianas deportistas nos hace suponer que la homosexualidad femenina en el deporte es atendida porque encaja con el discurso masculino del deporte. Históricamente las mujeres han sido disuadidas de evitar el deporte por la muscularización que conlleva y la relación de ésta con la masculinización de las mujeres (Giulianotti, 2011).

Si bien la orientación sexual no tiene incidencia en la conducta masculina o femenina de una persona, en el imaginario social a una mujer lesbiana se le adjudica una personalidad con carga masculina, lo cual se relaciona con las características masculinas de competitividad y fuerza que conlleva el deporte. Los hombres homosexuales, por el contrario, son adjudicados con una personalidad femenina, lo cual, en el imaginario, es contrario al espíritu deportivo, por sus características sociales de pasividad, debilidad y falta de competencia.

Lo anterior no debe suponer un mejor escenario para las mujeres en el deporte, el mismo, como institución, se encuentra altamente segregado por géneros (Anderson, Magrath et al, 2016) y aun y cuando se fomenta su participación, el deporte reproduce la masculinidad ortodoxa que ayuda a promover y perpetuar el patriarcado (Giulianotti, 2011).

Moscoso y Piedra (2019) en su texto El Colectivo LGBTI en el deporte como objeto de investigación sociológica. Estado de la Cuestión, precisan en el mismo sentido:

A pesar del cambio social experimentado en las sociedades avanzadas, el deporte sigue siendo uno de los pilares más acentuados de la dominación androcéntrica, con gran hostilidad hacia la presencia de gays y lesbianas en sus espacios de práctica, determinando estructural y simbólicamente la institución deportiva (pág. 501)

En lo que respecta a las metodologías y técnicas (Ver Tabla 3) identificadas en los estudios, al asumirse un asunto privado la determinación sexual, sumado, a la reducida investigación sobre la temática y escasos investigadores (Kozak, 2006) la presencia de investigaciones cualitativas es más alta. Por tanto, las técnicas como la entrevistas y análisis documentales, se vuelven los instrumentos para acceder a la información de pequeños grupos, lo que impide realizar generalizaciones sobre los resultados. 
TABLA 3

La representatividad por país, muestras, metodologías y técnicas de investigación utilizadas en los textos localizados.

\begin{tabular}{|l|l|l|l|}
\hline País & Población-muestra & Metodologías & Técnicas \\
\hline España (9) & Escolares nivel básico & Cuantitativa (2) & Entrevista (6) \\
Brasil (7) & Docentes nivel básico & Cualitativa (18) & Cuestionario (2) \\
México (2) & Universitarios & & Etnografia (2) \\
& Deportistas no Homosexuales & & Grupos focales (2) \\
& Mujeres deportistas lesbianas & & Mixtas (2) \\
& Afícionados homosexuales & & Análisis de discurso \\
& Deportistas olímpicosy & & (1) \\
& profesionales & & Documental(5) \\
\hline
\end{tabular}

Fuente: Elaboración propia.

En cuanto a los tipos y temáticas de los estudios (Ver Tabla 4), la mayor presencia encontrada en las bases de datos son los artículos y capítulos de libro, seguido por las tesis; así mismo fueron publicadas en revistas de ciencias de la actividad física, ciencias sociales y de estudios de género.

Finalmente, las principales temáticas abordadas en los textos revisados son: Actitudes hacía las personas homosexuales, comportamiento entre pares escolares en clase de EF, discriminación y exclusión, espacios y prácticas identitarias, así como, recuentos históricos y teóricos de la evolución y abordajes del tema en cuestión.

TABLA 4

Análisis sobre los ámbitos temáticos de los textos localizados por tipo de texto, publicaciones, abordajes y grupos de investigación.

\begin{tabular}{|l|l|}
\hline Tipo de Texto & $\begin{array}{l}\text { Tesis (4) } \\
\text { Artículos (12) } \\
\text { Capítulos de libro (3) } \\
\text { Ponencia Congreso Intemacional (1) }\end{array}$ \\
\hline Temática de publicaciones & $\begin{array}{l}\text { Revistas de Ciencias de la Actividad Fisica (7) } \\
\text { Revistas de Ciencias Sociales (7) } \\
\text { Revistas de Educación(2) } \\
\text { Revistas Estudios de géneros y repositorios (4) }\end{array}$ \\
\hline Autores con LGAC por país & $\begin{array}{l}\text { Vagner Matias Do Prado (Brasil) } \\
\text { Joaquín Piedra (España) }\end{array}$ \\
\hline Principales temáticas abordadas & $\begin{array}{l}\text { Actitudes hacía las personas homosexuales } \\
\text { Comportamiento entre pares escolares en clase de EF } \\
\text { Discriminación y exclusión } \\
\text { Espacios y prácticasidentitarias }\end{array}$ \\
\hline
\end{tabular}




\section{Conclusiones}

La aplicación práctica de este trabajo, está centrada en las oportunidades de los grupos de investigación para desarrollar líneas de generación y aplicación del conocimiento, así como, la inclusión de la temática en los programas de investigación con abordaje sociocultural, ello, permitirá el crecimiento de los estudios sobre deporte y homosexualidad y por ende, la publicación de trabajos en revistas indexadas, o bien, en editoriales de alto prestigio, para elevar las posibilidades de crecimiento del objeto de estudio en el mundo científico.

Tras la revisión sistemática se identifica que el cumulo de evidencia de divulgación científica es aún incipiente y con agendas de investigación apenas haciendo surgiendo. Es España y Brasil, los países que han profundizado en estas temáticas, lo que ha beneficiado, primeramente, en generar líneas de investigación en las Universidades y grupos de investigación y en un segundo término, que las aportaciones sean materiales de uso para las discusiones políticas y legislativas (como es el caso reciente en Brasil, sobre la discusión en el Congreso para la incorporación de los deportistas trans a las ligas deportivas de voleibol), que mejoren las condiciones del deporte diverso.

En prospectiva se sugiere continuar con los estudios de revisiones sistemáticas para en un futuro lograr compendiar los trabajos en meta análisis en el campo de las ciencias sociales, así como, búsquedas de materiales con otras categorías, en el idioma inglés y bases de datos, o revistas de paga, para ampliar la revisión bibliográfica.

\section{ReFERENCIAS}

Antúnez, M. (2009) Sexualidad y futbol. Dos resistencias femeninas en "Lesbianas de Buenos Aires". Memorias de la IJornadas del Centro Interdisciplinario de Investigaciones en Género, Universidad Nacional de La Plata. Facultad de Humanidades y Ciencias de la Educación. Instituto de Investigaciones en Humanidades y Ciencias Sociales (UNLP-CONICET). Centro Interdisciplinario de Investigaciones en Género. Tomado de: http://www.mem oria.fahce.unlp.edu.ar/library? $=\mathrm{d} \& \mathrm{c}=$ eventos\&d=Jev3893

Anderson, E., Magrath, R., \& Bullingham, R. (2016). Out in Sport: The experiences of openly gay and lesbian athletes in competitive sport. Milton Park, Abingdon, Oxon: Routledge.

Barreiro, A. (2004). La construcción social del cuerpo en las contemporáneas. Papers: Revista de sociología, 127-152. DOI: https://doi.org/10.5565/rev/papers/v73n0.1111

Baudrillard, J. (2004). El sistema de los objetos. México: Siglo XXI

Blanchard, K., \& Cheska, A. T. (1986). Antropología del deporte. México: Bellaterra.

Cavanagh, S., Sykes, H., \& Lamas, M. (2009). Cuerpos transexuales en las Olimpiadas: las políticas del Comité Internacional Olímpico en relación con 1@s atletas transexuales en los Juegos de Verano, Atenas 2004. Debate feminista, 39, 40-74. https://www.jstor.org/stable/42625543

Clarke, G. (1997). Reproducción de una parte: la vida de los profesores de educación física lesbianas. En G. Clarke \& B. Humberstone (Eds.), ResearchingWomen and Sport, Pág. 36-49. London: Macmillan

Clarke, G. (1998). Queering, el terreno de juego y salir a jugar: Lesbianas en la educación física y el deporte. Deporte, Educación y Sociedad, 3(2), 145-160.

De Sousa, V. G., \& De Camargo, W. (2015). 'Coligay' la diversidad sexual en el ámbito deportivo. Recorde, 8(1), 1. h ttps://revistas.ufrj.br/index.php/Recorde/article/view/2305

Fernández-Sánchez, H., King, K., \& Enríquez-Hernández, C. (2020). Revisiones Sistemáticas Exploratorias como metodología para la síntesis del conocimiento científico. Enfermería universitaria, 17(1), 87-94. Epub 22 de diciembre de 2020. https://doi.org/10.22201/eneo.23958421e.2020.1.697

Fuentes, M. (2015). Educación física y deporte en personas trans: una aproximación cualitativa. Tesis Doctoral. Universidad de Valencia, España. 
Franco, N. (2016). A Educação Física como território de demarcação dos gêneros possíveis: vivências escolares de pessoas travestis, transexuais e transgêneros. Motrivivência, 28(47), 47-66. https://doi.org/10.5007/2175-8042 $.2016 \mathrm{v} 28 \mathrm{n} 47 \mathrm{p} 47$

Garrido, J., \& Collado, N. (2018). Transgénero y Deporte. Revista Aranzadi de derecho de deporte y entretenimiento, (60), 5 .

Giddens, A. (1995). Modernidad e identidad del yo: el yo y la sociedad en la época contemporánea. Barcelona: Península. Giulianotti, R. (2011). Sport: A critical sociology. Cambridge: Political Press.

González Garzón, J. (2019). Educación física y deporte trans-formado. Praxis Educación y Pedagogía, (4). DOI: http s://doi.org/10.25100/praxis_educacion.v0i4.9121

Kozak, G. (2006). ¿Estudios sobre diversidad sexual, estudios sobre minorías sexuales? Revista venezolana de economía $y$ ciencias sociales, 12(3), 135-141. http://ve.scielo.org/scielo.php?script=sci_arttext\&pid=S1315-6411200600 0300008

Manchado Garabito, R., Tamames, S., López, M., Mohedano, L., D’Agostino, M. \& Veiga de Cabo, J. (2009). Revisiones sistemáticas exploratorias. Medicina y seguridad del trabajo, 55(216), 12-19. https://scielo.isciii.es/s cielo.php?pid=S0465-546X2009000300002\&script $=$ sci_arttext\&tlng=pt

Moioli, A., Machado, A., Zanetti, C., Campbell, F., \& Gomes, R. (2014). Soccer and homosexuality: The conflicts that lie within the affective game of the coach-adolescent athlete relationship. Motriz: Revista de Educação Física, 20(4), 346-358. https://doi.org/10.1590/S1980-65742014000400001

Moscoso, D. (2006). La sociología del deporte en España. Estado de la cuestión. Revista internacional de sociología, 64(44), 177-204. DOI: https://doi.org/10.3989/ris.2006.i44.33

Moscoso, D., \& Piedra, J. (2019). El colectivo LGTBI en el deporte como objeto de investigación sociológica. Estado de la cuestión. Revista Española de Sociología, 28(3). 501-516. https://dialnet.unirioja.es/servlet/articulo?codig $\mathrm{o}=7365737$

Pereira, A., Alfaia, A., Souza, L., \& Lima, T. (2014). Prejuicio contra homosexuales en el contexto de fútbol. Psicologia \& Sociedade, 26(3), 737-745. https://doi.org/10.1590/S0102-71822014000300022

Pérez, A., Arellano, A. \& Peña, C. (2013). Una búsqueda en Redalyc sobre diversidad sexual y adolescentes: implicaciones legales, metodológicas y sociales. En: Larios, J. \& De La Mora, J. La Diversidad Sexual en la Escuela Secundaria, pág. 19-31. Colima: SINED-UdeC.

Pérez-Aragón, P. \& Gallardo-Pérez, J. (2016). Thomas Arnold en la obra de Coubertin. El mito de Arnold. Ágora para la educación fisica y el deporte, 18(1), 20-35. http://agora-revista.blogs.uva.es/files/2016/05/agora_18_1b_pere z_et_gallardo.pdf

Piedra, J., Macías, R., \& Romero, A. L. (2014). Visibilizando lo invisible: creencias del profesorado de educación física sobre homofobia y masculinidades. Retos: nuevas tendencias en educación fisica, deporte y recreación, (25), 36-42. http://hdl.handle.net/11441/39659

Piedra, J. (2015a). D. Homosexualidad y deporte, ¿ un matrimonio mal avenido?. En Biofanex La visión integradora de la investigación en ciencias del deporte, 26-33. Editorial Universidad de Sevilla.

Piedra, J. (2015b). Gays y lesbianas en el deporte: discurso de jóvenes universitarios españoles en torno a su aceptación. Movimento. Revista da scola de educaçao física UFRGS, 21(4), 1067-1081. https://www.redalyc.org/pdf/1153/ 115343227017.pdf

Piedra, J. (2016). Escala de Actitudes hacia la Diversidad Sexual en el Deporte (EDSD): desarrollo y validación preliminar. Revista de psicologia del deporte, 25(2), 299-307. https://www.redalyc.org/pdf/2351/23514651501 1.pdf

Piedra, J. (2019). La perspectiva de género en sociología del deporte en España: presente y futuro. Revista Española de Sociología, 28(3). 489-500. https://dialnet.unirioja.es/servlet/articulo?codigo=7365737

Pontes De Los Ríos, M. (2019). Abriendo armarios. Reportaje ciberperiodistico sobre la realidad de los deportistas homosexuales en España. Tesis de Grado. Universidad de Valladolid. 
Ramírez, A. (2014). Mujeres que" sexúan" con mujeres. Experiencia sexual y deportiva entre futboleras de Morelos. Tesis Maestría. México, DF: CIESAS.

Rodríguez, R. (1998). Fútbol y homosexualidad (un deporte para machos). Alabarces, P.; Di Giano, R. y Frydenberg, J.(comps.) Deporte y Sociedad, Buenos Aires, Eudeba.

Salazar, C., Arellano, A., Pérez, A \& Manzo, G. (2014). Jóvenes y ocio activo orientado a la actividad física y el deporte. Una revisión a las publicaciones de la Red de Revistas Científicas de América Latina y el Caribe, España y Portugal. En: Martínez, S. \& Peralta, R (comps.) Estudios sobre Ocio y Recreación. Abordajes Críticos desde Argentina, Brasil, Colombia y México. Pp 68-81. México D.F: INDECUS.

Salazar, C. \& Manzo, G. (2016) La voz del cuerpo, prejuicios de la vista y práctica deportiva. En Larios, J. \& De la Mora. J., en Diversidad sexual y universidad, 27-52. Universidad de Colima, México.

Sagarzazu, I. \& Lallana, I. (2012). Estrategias del comité olímpico internacional para la igualdad de género en el deporte y la imagen mediática de las deportistas. In Libro de Actas del I Congreso Internacional de Comunicación y Género. (pp. 2048-2068). Sevilla: Facultad de Comunicación. Universidad de Sevilla.

Silva, P., Jaeger, A. \& Valdivia-Moral, P. (2018). Percepción de los Estudiantes sobre Comportamientos Homofóbicos y Heterosexistas en Educación Física. Revista de Psicología del Deporte. https://revistes.uab.cat/rpd/article/vie w/v27-n2-silva-jaeger-valdivia-etal

Stigger, M \& Da Silveira, R. (2010). Ocio y homosexualidad: un estudio etnográfico sobre el asociativismo deportivo de mujeres, en el contexto de un deporte dicho masculino. Polis Revista de la Universidad Bolivariana, Vol.9, Núm. 26. 133-155. https://journals.openedition.org/polis/174

Organización de las Naciones Unidas para la Educación, la Ciencia y la Cultura (2017). Guía para asegurar la inclusión y la equidad en la educación. Francia, Francia: UNESCO.

Vagner, M. (2017). Entre queerpos e discursos: normalização de condutas, homossexualidades e homofobia nas práticas escolares da Educação Física. Práxis Educativa, 12(2), 501-519. https://dialnet.unirioja.es/servlet/arti culo?codigo $=6046860$ 